J. DIFFERENTIAL GEOMETRY

53 (1999) $177-182$

\title{
EIGENFUNCTIONS WITH FEW CRITICAL POINTS
}

\author{
DMITRY JAKOBSON \& NIKOLAI NADIRASHVILI
}

\begin{abstract}
We construct a sequence of eigenfunctions on $\mathbf{T}^{2}$ with a bounded number of critical points.
\end{abstract}

S. T. Yau raised a question about the number and distribution of critical points of eigenfunctions of the Laplacian on a Riemannian manifold ([4, \# 76], [5, \# 43]). In [6] he investigated this problem in two dimensions and proved, in particular, that under certain curvature assumptions every eigenfunction has a critical point where the critical value is uniformly bounded. Here we prove

Theorem 1. There exists a metric on the two-dimensional torus and a sequence of eigenfunctions such that the corresponding eigenvalues go to infinity but the number of critical points remains bounded.

This answers in the negative the question raised in [4]; however, our metric is quite special, and it is possible that for a generic metric the number of critical points increases with the growth of the eigenvalue.

The main idea of our construction is to consider a sequence of eigenfunctions $f_{n}(x, y)=\sin (n x+y)$ (on $\mathbf{T}^{2}$ with the flat metric) whose critical points lie on a union of two line segments, and then change a metric in such a way that instead of two critical "ridges" we shall have a bounded number of critical points.

We consider a Liouville metric (cf. [3])

$$
q(x)\left(d x^{2}+d y^{2}\right)
$$

Received December 7, 1999. The first author was partially supported by NSF grant DMS-9802947, and the second author by NSF grant DMS-9971932.

Key words and phrases. Laplacian, eigenfunction, critical point, WKB

1991 Mathematics Subject Classification. 34E20, 58J50 
on the torus $\mathbf{T}^{2}=\{(x, y): 0 \leq x, y \leq 2 \pi\}$. Here $q$ is a smooth periodic function whose properties we shall specify later. Joint eigenfunctions of the Laplacian $\Delta=(1 / q(x))\left(\partial^{2} / \partial x^{2}+\partial^{2} / \partial y^{2}\right)$ and $\partial / \partial y$ have the form

$$
f(x, y)=\varphi(x) e^{i m y}, \quad m \in \mathbf{Z},
$$

where $\varphi$ satisfies an equation (cf. $[3,(4.3)]$ )

$$
\varphi^{\prime \prime}(x)+\left(\lambda q(x)-m^{2}\right) \varphi(x)=0 .
$$

In the rest of the paper we shall choose $m=1$. Accordingly, $\varphi$ satisfies

$$
\varphi^{\prime \prime}(x)+(\lambda q(x)-1) \varphi(x)=0 .
$$

We choose $q$ to be a periodic function of period $\pi / 2$ and let $\varphi$ satisfy (2) on $[0, \pi / 2]$ with boundary conditions

$$
\varphi^{\prime}(0)=\varphi(\pi / 2)=0 .
$$

Then the function $\varphi_{1}$ defined by

$$
\varphi_{1}(x)= \begin{cases}\varphi(x), & x \in[0, \pi / 2], \\ -\varphi(\pi-x), & x \in[\pi / 2, \pi], \\ -\varphi(x-\pi), & x \in[\pi, 3 \pi / 2], \\ \varphi(2 \pi-x), & x \in[3 \pi / 2,2 \pi] .\end{cases}
$$

and its shift $\varphi_{2}$ defined by

$$
\varphi_{2}(x)=\varphi_{1}(x+\pi / 2)
$$

are two linearly independent solutions of $(2)$ on $[0,2 \pi]$ (we are considering $x \bmod 2 \pi$ and using the periodicity of $q$ ).

We denote the spectrum of (2) on $[0, \pi / 2]$ with boundary conditions (3) by $0<\lambda_{1}<\lambda_{2}<\ldots$ Then every $\lambda_{j}$ is an eigenvalue of multiplicity two of the equation (2) on $[0,2 \pi]$ with periodic boundary conditions (the corresponding eigenfunctions $\varphi_{1,2}(j)$ are given by (4) and (5)). We next investigate the function $g_{j}(x)$ defined by

$$
g_{j}(x)=\varphi_{1}(j)(x)^{2}+\varphi_{2}(j)(x)^{2} .
$$

Lemma 2. There exists $C>0$ such that for $\lambda_{j}$ large enough the function $g_{j}(x)$ is monotonic outside the union of $\left(C / \lambda_{j}\right)$-neighborhoods of the critical points of $q(x)$. 
Proof. The solutions $\psi_{j}(x)=\varphi_{1}(j)(x)+i \varphi_{2}(j)(x)$ of the equation (2) can be asymptotically expanded in $t=\sqrt{\lambda}$ (cf. [1], [2, p. 34]). We can make a change of variable (cf. [2, p. 32])

$$
\psi(x)=\exp \left\{d_{j} \int_{0}^{x} \sum_{k=-1}^{\infty} t^{-k} \alpha_{k}(s) d s\right\}
$$

in the equation

$$
\psi^{\prime \prime}+\left(t^{2} q-1\right) \psi=0
$$

Here $\varphi_{j}(0)=1$ and

$$
d_{j}=\frac{\psi_{j}^{\prime}(0)}{\sum_{k=-1}^{\infty} t_{j}^{-k} \alpha_{k}(0)}
$$

is the normalization constant.

Further substitution $\psi^{\prime} / \psi=w$ reduces the equation above to the Ricatti equation

$$
w^{\prime}+w^{2}+t^{2} q(x)-1=0
$$

for $w=\sum_{k=-1}^{\infty} t^{-k} \alpha_{k}(x)$ from which $\alpha_{k}$-s can be found inductively from the asymptotic expansion in $t$.

In particular, $\alpha_{-1}^{2}+q=0$. We assume that $q(x)$ is not identically constant and that

$$
q(x) \gg 1
$$

so we can choose

$$
\alpha_{-1}(x)=i \sqrt{q(x)}
$$


The next few terms are given by

$$
\begin{aligned}
& \left\{\begin{array}{l}
\alpha_{0}=-q^{\prime} /(4 q) \\
\alpha_{1}=(-i)\left(1 /\left(2 q^{1 / 2}\right)-5\left(q^{\prime}\right)^{2} /\left(32 q^{5 / 2}\right)+q^{\prime \prime} /\left(8 q^{3 / 2}\right)\right)
\end{array}\right. \\
& \alpha_{2}=\left(q^{\prime \prime \prime}-4 q^{\prime}\right) /\left(16 q^{2}\right)-9 q^{\prime} q^{\prime \prime} /\left(32 q^{3}\right)+15\left(q^{\prime}\right)^{3} /\left(64 q^{4}\right), \\
& \alpha_{3}=\frac{-i}{8 q^{3 / 2}}\left(1+\frac{6 q^{\prime \prime}-q^{\prime \prime \prime \prime}}{4 q}+\frac{28 q^{\prime} q^{\prime \prime \prime}+19\left(q^{\prime \prime}\right)^{2}-50\left(q^{\prime}\right)^{2}}{16 q^{2}}\right. \\
& \left.+\frac{1105\left(q^{\prime}\right)^{4}}{256 q^{4}}-\frac{221\left(q^{\prime}\right)^{2} q^{\prime \prime}}{32 q^{3}}\right) \\
& \alpha_{4}=\frac{q\left(q^{\prime \prime \prime \prime \prime}-8 q^{\prime \prime \prime}+16 q^{\prime}\right)+17 q^{\prime \prime} q^{\prime \prime \prime}+10 q^{\prime} q^{\prime \prime \prime \prime}-54 q^{\prime} q^{\prime \prime}}{64 q^{4}} \\
& +\frac{3 q^{\prime}\left(80\left(q^{\prime}\right)^{2}-102\left(q^{\prime \prime}\right)^{2}-75 q^{\prime} q^{\prime \prime \prime}\right)}{256 q^{5}} \\
& +\frac{1695\left(q^{\prime}\right)^{3}\left(2 q q^{\prime \prime}-\left(q^{\prime}\right)^{2}\right)}{1024 q^{7}}
\end{aligned}
$$

Let $h_{j}(x)$ be a constant multiple of the logarithmic derivative of the function $g_{j}(x)=\psi_{j}(x) \overline{\psi_{j}(x)}$,

$$
h_{j}(x)=\frac{g_{j}^{\prime}(x)}{2 d_{j} g_{j}(x)} .
$$

It has an asymptotic expansion in $\lambda_{j}=t_{j}^{2}$ given by

$$
h_{j}(x)=\sum_{k=0}^{\infty} \alpha_{2 k}(x) \lambda_{j}^{-k}
$$

The error term in the $n$-term expansion is $O\left(\lambda_{j}^{-n}\right)$, uniformly in $x$ and $j$ (cf. [1], [2]). The lemma now follows from (7) and (8). q.e.d.

We next investigate the behavior of $g_{j}(x)$ in the $C / \lambda_{j}$-neighborhoods of the critical points of $q(x)$. We assume that $q(x)=q(\pi / 2-x)$ and that $q$ has a unique minimum at 0 and a unique maximum at $\pi / 4$ on $[0, \pi / 2)$. The Taylor expansion of $q$ at a critical point $x_{0}$ has the form

$$
q\left(x_{0}+x\right)=a_{0}\left(1+a_{1} x^{2}+\sum_{j=2}^{\infty} a_{j} x^{2 j}\right),
$$

where $a_{0}>0, a_{1}>0$ at $x_{0}=0$ and $a_{0}>0, a_{1}<0$ at $x_{0}=\pi / 4$. It follows from the symmetries of $q$ that $g_{j}(x)=g_{j}(-x), g_{j}(\pi / 4-x)=g_{j}(\pi / 4+x)$. 
We next differentiate (8) (cf. [1], [2]) and substitute (9) into the resulting expression to study the asymptotic expansions of $h_{j}^{\prime}(x)=h^{\prime}(x)$ (in $x$ and $\lambda_{j}=\lambda$ ) in $C / \lambda_{j}$-neighborhoods of $x_{0}=0$ and $x_{0}=\pi / 4$. We get

$$
h^{\prime}\left(x_{0}+x\right)=b_{1}(\lambda)+b_{2}(\lambda) x^{2}+O\left(|x|^{4}+\lambda^{-3}\right),
$$

uniformly in $j$; here

$$
\left\{\begin{aligned}
b_{1}= & \frac{-a_{1}}{2}+\frac{1}{\lambda a_{0}}\left[\frac{3 a_{2}-a_{1}}{2}-\frac{9 a_{1}^{2}}{8}\right] \\
& +\frac{1}{\left(\lambda a_{0}\right)^{2}}\left[3 a_{2}-\frac{2 a_{1}+45 a_{3}}{4}+\frac{a_{1}\left(324 a_{2}-54 a_{1}-153 a_{1}^{2}\right)}{16}\right] \\
\frac{b_{2}}{3}= & \frac{a_{1}^{2}}{2}-a_{2}+\frac{1}{\lambda a_{0}}\left[a_{1}^{2}-a_{2}-12 a_{1} a_{2}+\frac{30 a_{3}-21 a_{1}^{3}}{4}\right] \\
& +\frac{1}{\left(\lambda a_{0}\right)^{2}}\left[15\left(a_{3}-7 a_{4}\right)-a_{2}+\frac{3 a_{1}^{2}}{2}+21 a_{1}^{3}+\frac{3225 a_{1}^{4}}{32}\right. \\
& \left.+\frac{3}{4}\left(122 a_{2}^{2}-48 a_{1} a_{2}-399 a_{1}^{2} a_{2}+280 a_{1} a_{3}\right)\right] .
\end{aligned}\right.
$$

The function $q(x)$ was chosen so that $a_{1} \neq 0$ in (9). It follows that in $C / \lambda_{j}$-neighborhoods of the critical points

$$
h_{j}^{\prime}\left(x_{0}+x\right)=\frac{-a_{1}}{2}+O\left(1 / \lambda_{j}\right)
$$

If $g_{j}$ had two or more critical points in a $C / \lambda_{j}$-neighborhood of a critical point of $q$, then $h_{j}$ would have at least two zeros there and $h_{j}^{\prime}$ would vanish, contradicting (11) for large enough $\lambda_{j}$. Therefore $g_{j}$ has at most one critical point in every such neighborhood for large $\lambda_{j}$. Together with Lemma 2 this proves

Lemma 3. The number of critical points of $g_{j}(x)$ is uniformly bounded above.

We are now ready to prove the theorem. Let

$$
f_{j}(x, y)=\varphi_{1}(j)(x) \sin y+\varphi_{2}(j)(x) \cos y,
$$

where $\varphi_{1,2}(j)(x)$ are defined by (4) and (5). The function $f_{j}(x, y)$ is equal to

$$
\left(g_{j}(x)\right)^{1 / 2} \sin \left(\Phi_{j}(x)+y\right)
$$


where $\Phi_{j}(x)$ is a continuous monotone function defined by

$$
\cos \left(\Phi_{j}(x)\right)=\varphi_{1}(j)(x) /\left(g_{j}(x)\right)^{1 / 2}, \sin \left(\Phi_{j}(x)\right)=\varphi_{2}(j)(x) /\left(g_{j}(x)\right)^{1 / 2}
$$

( $\Phi$ is monotone since a nonzero linear combination of $\varphi_{1}$ and $\varphi_{2}$ cannot have a second order zero).

At a critical point $\left(x_{0}, y_{0}\right)$ of $f_{j}$ we have

$$
\frac{\partial f_{j}}{\partial y}=\left(g_{j}(x)\right)^{1 / 2} \cos \left(\Phi_{j}(x)+y\right)=0
$$

so

$$
y+\Phi_{j}(x)=\pi / 2+\pi k, k \in \mathbf{Z} .
$$

Also,

$$
\frac{\partial f_{j}}{\partial x}=\frac{g_{j}^{\prime}(x)}{2\left(g_{j}(x)\right)^{1 / 2}} \sin \left(\Phi_{j}(x)+y\right)=0
$$

(we have used the equality $\cos \left(\Phi_{j}(x)+y\right)=0$ ). Accordingly, by Lemma $3, x$ can take a bounded number of values. Together with (13) this shows that the number of critical points of $f_{j}(x, y)$ is uniformly bounded above, and the proof is finished. q.e.d.

Remark. One can show that for large $\lambda_{j}$ the eigenfunctions that were constructed have exactly 16 critical points.

\section{References}

[1] M. Evgrafov \& M. Fedoryuk, Asymptotic behavior of solutions of the equation $w^{\prime \prime}(z)-p(z, \lambda) w(z)=0$ as $\lambda \rightarrow \infty$ in the complex $z$-plane, Russian Math. Surveys 21 (1966) 1-48.

[2] M. Fedoryuk, Asymptotic analysis: linear ordinary differential equations, Springer, Berlin, 1993.

[3] D. Kosygin, A. Minasov \& Y. Sinai, Statistical properties of the spectra of LaplaceBeltrami operators on Liouville surfaces, Russian Math. Surveys 48 (1993) 1-142.

[4] S. T. Yau, Problem section, Seminar on Differential Geom., (ed. by S. T. Yau) Ann. of Math. Stud. 102 (1982) 669-706.

[5] — Open problems in geometry, Differential geometry: partial differential equations on manifolds, Proc. Symp. Pure Math. 54 (1993) 1-28.

[6] - A note on the distribution of critical points of eigenfunctions, Tsing Hua Lectures in Geometry and Analysis 315-317, Internat. Press, 1997.

UNIVERsity of ChICAGo 